\title{
La Comédie humaine
}

\section{une réponse}

\section{Hans R. Runte}

"Ce n'est pas une vue bourgeoise du monde réel, c'est une vue réaliste du monde bourgeois."I

Balzac n'est pas universellement aimé. Aussi 1'amour que lui porte 1'auteur de notre aphorisme est-il bien un amour de tête. Car M. Wurmser est trop subtil (ou trop sincèee) pour dire tout nettement avec Maxime Nemo: "Je hais Balzac."2 Comment aussi aurait-il pu faire d'un livre aussi "humain"3 que 1 'est La Comédie inhumaine un livre qui suscite la haine?

Il faut donc différencier. M. Wurmser, lui non plus n'aime pas Balzac, mais c'est 1 'homme qu'il hait. I1 adore 1'écrivain. Il adore le monde balzacien parce qu'il y trouve d'innombrables illustrations de sa propre idéologie sociale.

Il déteste Balzac le bourgeois, et il n'aime le romancier réaliste que par les raisons qu'il lui procure de détester la bourgeoisie.

Le réalisme tel que le comprend $M$. Wurmser est un réalisme vu à travers une idéologie. C'est pourquoi La Comédie Humaine ne peut être "une vue bourgeoise du monde réel", la critique de l'oeuvre se réduirait alors à un pamphlet biographique, mais doit être le "monde bourgeois" détestable par principe, vu par un "réaliste", qui a le malheur d'être un bourgeois.

Il est évident que la notion wurmserienne du réalisme, prisonnière d'une idéologie, est trop étroite pour s'appliquer à la totalité de la création balzacienne. La réalité de La Comédie Humaine n'est pas exclusivement celle de 1 'or.

Pour M. Wurmser, le "monde réel" est "1e monde bourgeois." Le mérite de Balzac serait d'avoir fait de la bourgeoisie non pas l'objet purement littéraire d'un 
roman "bourgeois," mais 1'objet sociologique d'une critique "réaliste," écrite, il est vrai, par un bourgeois mais qui est d'une lucidité exceptionnelle.

Qu'on puisse voir, dans les personnages de La Comédie Humaine, des bourgeois qui tournent autour de $1^{\prime}$ or, soit. Mais le réalisme de Balzac ne s'y arrête pas.

La danse des personnages autour du veau d'or révèle une sous-réalité qui n'est plus bourgeoise, mais grotesque.

Je m'explique. Le grand fleuve de la création réaliste, qui jaillit de la source Saint-Hilaire et se jette dans 1'océan de La Comédie Humaine, coule majestueusement entre les bords de 1'analyse psychologique et de la critique socio-morale, tout en emportant avec lui les eaux plus ou moins troublées de ses tributaires romantiques ou idéalistes.

En effet, derrière l'inchangeable thème balzacien du jeune ambitieux se cache, dans Le Père Goriot, I'idéalisme du "Christ de la paternité," dans Le Lys dans la Vallée, la "Vierge de la maternité." Dans Illusions perdues coulent les flots souterrains d'un amour romantique qui rejaillit dans Splendeurs et Misères des Courtisanes, roman qui, par ailleurs, accumule de romanesques enlèvements, attentats, et déguisements.

Examinons quelques-unes des scènes où le sous-réel grotesque monte à la surface et devient visible.

Le Père Goriot est une oeuvre des "temps de douloureuse littérature, "4 autant vaut dire une oeuvre larmoyante: "l'oeuvre accomplie, peut-être aura-t-on versé quelques larmes..."(18). La scène est "entre les buttes de Montmartre et les hauteurs de Montrouge, dans cette illustre vallée de platras incessament près de tomber et de ruisseaux noirs de boue"(18) où 1'"obscure, mais effroyable tragédie parisienne"(149) se jouera dans "un océan de boue"(384). Le décor est lugubre: ".... rue Neuve-Sainte-Geneviève... Ie jour diminue et le chant du conducteur se creuse, alors que le voyageur descend aux Catacombes. ' Comparaison vraie! Qui décidera de ce qui est plus horrible à voir, ou des coeurs desséchés, ou des crânes vides?(20)... il s'y rencontrera toujours un lieu vierge, un antre inconnu, des fleurs, des perles, des monstres, quelque chose d'inoui, oublié par les plongeurs littéraires. La maison Vauquer est une de ces monstruosités ${ }^{5}$ curieuses"(33). Aussi 
Balzac s'est-il plongé avec délices dans cette mer d'où il tire au jour ce misérable père Goriot qui saisit les paroles de ses filles "comme un chien saisit un mouvement de son maître"(215). Ses sentiments paternels touchent à la démesure quand il apprend la misère de Delphine: "Mon Dieu! pleurer, elle a pleuré? -La tête sur mon gilet, dit Eugène. -Oh! donnez-le-moi"(237). Peu avant sa mort, son dévouement devient sans borne: "Mais on ne peut donc rien faire de son sang? cria le vieillard désespéré (365)...je serai plus qu'un père pour toi, je veux être une famille! Je voudrais être Dieu, je te jetterais 1'univers aux pieds" (367). Si de telles éruptions monstrueuses ont peut-être encore quelque chose de sublime, d'autres personnages, pêchés, eux aussi, dans "1'océan de boue," sont simplement macabres. Gobseck est caractérisé par une seule phrase saillante: il est "capable de faire des dominos avec les os de son père"(75). Vautrin, lui, est plus complexe, mais il a certainement un côté gigantesque:

"souvent il laissait percer l'épouvantable profondeur de son caractère (38)...cet homme, qui ne fut plus un homme, mais le type de toute une nation dégénérée, d'un peuple sauvage et logique, brutal et souple. En un moment Collin devint un poème infernal... Son regard était celui de 1'archange déchu"(311). A côté d'un tel homme, les autres paraissent des "esprits lilliputiens" (51), et cette allusion achève $1^{\prime}$ impression d'un monde fantastique que Balzac a trouvé au fond de la mer parisienne.

Une analyse stylistique du Lys dans la Vallée démontrerait qu'en matière romantique, Balzac était plus catholique que le pape. On comprendrait parfaitement pourquoi il se disait, par exemple, admirateur de Lamartine ${ }^{6}$. Je me bornerai ici à ne relever que quelquesuns des traits les plus marquants.

Natalie, dans la lettre finale du roman, résume 1 'oeuvre par ce mot révélateur: "je ne sais rien de plus monstrueux"7 (357). En effet, rien de plus violent, par exemple, que la première rencontre de Félix avec Madame de Mortsauf: "je me plongeai dans ce dos comme un enfant qui se jette dans le sein de sa mère, et je baisai toutes ces épaules en y roulant ma tête"(35). Cette explosion d'animalité se changera peu après en 
effusions sentimentales qui deviennent parfois écoeurantes: "Deux grosses larmes éclairées par un rayon de lune sortirent de ses yeux, roulèrent sur ses joues, en atteignirent le bas; mais je tendis la main assez à temps pour les recevoir, et les bus avec une avidité pieuse.. .. -Voici, lui dis-je, la première, la sainte communion de 1'amour. Oui, je viens de...m'unir à votre âme, comme nous nous unissons au Christ en buvant sa divine substance"(103). Le thème de la communion de l'amour revient à plusieurs reprises, sous les noms d'abord de fiançailles d'âmes $(73,325)$, puis de mariage d'âmes $(93,120,228,230,249,280)$. A ce symbolisme qui frôle le mauvais goût se joignent des comparaisons avec Dieu, déjà signalées dans Le Père Goriot. Voici comment Arabelle parle de 1'amour: "soumettre le monde et le ciel à un homme en dérobant ainsi au Tout-Puissant le droit de faire un Dieu... Voilà des grandeurs où n'atteignent pas les femmes vulgaires" (251). Ou bien 1'allusion de Félix lorsqu'il est auprès de Madame de Mortsauf: "tout ce que je puis avoir de grand en moi vient de vous. Ne savez-vous donc plus que je suis votre ouvrage?" (273). La scène à Clochegourde n'est pas moins lugubre lors de la maladie de M. de Mortsauf et de la mort de sa femme. Félix entre plus avant dans le coeur de son Henriette, et ce "fut une scène d'enfance à côté d'une tombe entr'ouverte" (228). Plus tard, quand Henriette est morte, Félix de s'écrier: "Ah! je 1'aimais morte, autant que je l'aimais vivante" (335). Peu de réalisme dans ces épisodes, peu de réalisme aussi dans les remarques très directes sur les caractères des trois personnages principaux. La passion de Félix "recommençait le Moyen Age et rappelait la chevalerie" (249). L'amour de Madame de Mortsauf fait lamenter Félix: "Si elle m'avait aimé, elle aurait eu pour mes souffrances cette expression de lionne au désespoir" (221). Cette lionne, Arabelle l'est parfaitement (250); et voilà Balzac au seuil de I'exotisme: "L'atonie que mettent dans $I^{\prime}$ existence de ces femmes une perfection constante dans les choses, une régularité méthodique dans les habitudes, les conduit à 1 'adoration du romanesque $^{8}$ et du difficile (245)...Aussi sa passion estelle tout africaine; son désir va comme le tourbillon du désert,...le désert plein d'azur et d'amour, avec... 
ses fraîches nuits étoilées" (247).

De semblables éléments ne se trouvent que rarement dans Illusions perdues. I1 se peut que le thème très actuel de la critique du journalisme, joint au thème directeur de l'ascension d'un jeune ambitieux, en ait rendu 1'élaboration plus concise et ait tracé des $1 i$ mites plus étroites à l'inspiration de l'auteur.

S'il y a du grotesque dans ce roman, les scènes où il est visible restent toujours au service du but réaliste de l'oeuvre. Il en résulte des saillies de comparaison qui révèlent le grand écrivain. Toute la description de la noblesse de province est comprise dans ce bout de phrase: "ces vieilles familles perchées sur leur roche de la haute ville d'Angoulême. comme des corbeaux défiants" (41); et le provincial qui vient d'arriver à Paris n'est qu'un "singulier jeune homme qui a l'air d'un mannequin habillé à la porte d'un tailleur" (171). Au symbolisme animal, déjà relevé dans Le Père Goriot (le chien) et Le Lys dans la Vallée (la lionne), nous devons cette apostrophe de De Marsay: "1e lion parisien laissa retomber son lorgnon si singulièrement qu'il semblait à Lucien que ce fût le couteau de la guillotine" (178). Cependant, le lugubre n'en est pas complètement absent. La scène de la mort de Coralie n'est-elle pas une des plus cruelles? "Lucien revint chez lui: il y trouva Coralie étendue droite et roide sur un lit de sangle... le poète se mit à sa table, auprès du corps de sa pauvre amie, et y composa les dix chansons qui voulaient des idées gaies et des airs populaires...Quelle nuit que celle où ce pauvre enfant se livrait à la recherche de poésies à offrir aux Goguettes en écrivant à la lueur des cierges, à côté du prêtre qui priait pour Coralie?" (440) Non pas macabre, mais grotesque, elle aussi, la nuit de la sérénade à Angoulême: "Après...trois salves, criées par quelques voix, trois couronnes et des bouquets furent adroitement jetés par la croisée dans 1'appartement... Le succès du grand homme de province était trop antipathique aux moeurs immobiles d'Angoulême pour ne pas avoir été mis en scène par des intérêts ou par un machiniste passionné, collaborations également perfides" (547-548).

Si Balzac s'est abstenu, dans Illusions perdues, de trop de monstruosités, il en comble, pour rattrapper 
son rival Eugène Sue, une de ses dernières oeuvres, les gigantesques Splendeurs et Misères des Courtisanes. Ce roman ne reprend qu'au second plan la grande idée ba1zacienne du jeune homme qui veut faire fortune, et traite, en précurseur du roman-policier, le combat souterrain que se livrent deux partis également clandestins. "Infernal" y est l'épithète dominant. Balzac n'aurait-il pas été conscient de l'ironie tournée contre lui-même quand il écrivait: "Après avoir acheté la vie de Lucien... Collin lui avait proposé 1 'un de ces pactes infernaux 9 qui ne se voient que dans les romans" (103), même s'il inwoque les procès-verbaux des Assises pour se justifier? De même trouve-t-on "dans les romans" seulement "une ardoise sur laquelle... Peyrade trouvait parfois des marques bizarres... Cette espèce d'algèbre infernale offrait aux initiés des significations très claires" (145). Aussi "1'activité vraiment infernale" d'Asie ne peut-elle empêcher le suicide de Lucien. Dans $1^{\prime}$ affreux ${ }^{10}$ univers balzacien les anges côtoyent les monstres. "Esther...n'était plus une courtisane, mais un ange qui se relevait d'une chute" (56). Et Collin, ce "dompteur de bêtes féroces" (84), dit d'elle avec une cruauté satanique: "Eh bien, isther est un gibier après lequel je vais faire courir... Nucingen." -Esther ne voudra jamais, dit Lucien. -Ça me regarde. -Elle en mourra. - Ça regarde les Pompes funèbres" (101). L'adversaire de Collin n'est pas moins monstrueux: "Le crâne... avait 1 'insensibilité d'une tête de mort, et, sans quelques cheveux à $1^{\prime}$ arrière, on eût doûté qu'il fût celui d'un homme vivant" (127). Aussi macabre que cette description est la scène du suicide de Lucien quand Collin arrive "à la cellule où gisait Lucien, qu'on avait mis sur le lit. A cet aspect, il tomba sur ce corps et s'y colla par une étreinte désespérée... -Laissez-moi 1à!...dit Jacques Collin d'une voix éteinte, je n'ai pas longtemps à le voir, on va me l'enlever pour... Il s'arrêta sur le mot enterrer" (477). $C$ 'est dans ce demi-monde de demi-vivants et de diables humains que se déroulent les tentatives de suicide, les enlèvements et escroqueries, les espionnages et déguisements, les meurtres et arrestations, les procès et, finalement, les suicides accomplis. 
Revenons à présent à M. Wurmser. Si La Comédie Humaine était "une vue bourgeoise du monde réel," ne seraient morts ni le père Goriot, ni Madame de Mortsauf, ni Lucien de Rubempré. Mais en les laissant périr par les forces déstructrices inhérentes à la bourgeoisie, Balzac fait oeuvre de critique, il présente "une vue réaliste du monde bourgeois."

Interprétation possible, et non des moins valables. Mais elle est aussi étroite que le monde balzacien est vaste. Ou autrement dit: elle ne s'applique, en naissant d'un système, qu'au système de Balzac.

Or, tout système ampute la réalité. Aussi l'opinion de M. Wurmser comme les théories de Balzac ne sont-elles qu'une vue partielle de la réalité de La Comédie Humaine. Balzac lui-même semble en avoir été plus conscient que ne l'est son critique. Car dans les passages que je viens de citer, il a prodigué tous ses efforts pour échapper à une société qu'il s'est imposé lui-même de décrire. Peut-être le véritable romancier se cache-t-il dans ces "digressions" où il crée les Goriot, les Mortsauf, les Coralie et les Esther.

Autant pour le "monde bourgeois."

Quant à la "vue réaliste," Balzac la garde même pendant ses excursions dans les mondes du grotesque et du monstrueux. Il n'abandonne ni la description soigneuse ni 1'analyse précise de ses anges et ses diables. Ces personnages "inhumains" sont aussi "vrais" que les autres comédiens de 1 'humanité. S'ils nous paraissent monstrueux, c'est par leur réalisme même: la monstruosité est l'effet d'un manque de différenciation de la part du créateur qui décrit ses êtres extraordinaires avec les mêmes moyens que les habitants de son système. Le réalisme, propre à Rastignac ou à Nucingen, mène chez Madame de Mortsauf ou le père Goriot à un décalage trop marqué entre forme et contenu pour que le goût du lecteur n'en soit pas choqué.

Avant de déclarer Balzac poète baroque, j'arrête en complétant $1^{\prime}$ avis de $M$. Wurmser dans ce sens:

La Comédie Humaine, "ce n'est pas une vue bourgeoise du monde réel, c'est une vue trop réaliste du monde inhumain." 
1 André Wurmser, "Variations sur la sincérité de Balzac," dans Europe, no. 429-430 (janvier-février 1965) p. 231 Maxime Nemo, "
$429-430$, pp. $324-325$

3 Maurice Regard, "Un gros volume qui se dévore avec passion", dans Europe, no. 429-430, p. 271

4 p. 17; toutes les citations des romans étudiés renvoient aux éditions du "Livre de Poche" et seront indiquées désormais dans le texte.

5 C'est moi qui souligne

6 cf. la dédicace de César Birotteau

7 C'est moi qui souligne

8 C'est moi qui souligne

9 C'est moi qui souligne

10 cf. 1'article de Pierre Citron, "Les affreux du miroir," dans Europe, no. 429-430 (janvier-février 1965) pp. 94-104




Te souviens-tu?

Est-ce que tu te souviens . . .

Vaguement de la mer de

la source sale et. . .

Te souviens-tu?

Est-ce que tu te souviens ...

Du sable ou les traces déçues

de nos améres (mal dériveśs) qui

sont, par le reflux de la mar emportees comme des cygnes

mal armés...

Non?

Tu es sans merci . . .

Un césarien avant Macbeth

Un edelweiss sans Montaigne

(Que ses jeux sont déRacinés!)

Encore non?

Tu ne ses pas que

l'immortaliste ne gît plus

$\underset{\mathbf{S}}{\mathbf{a}}$

ou moins que de puits

la grande sécheresse

(le contraire de nos éclairs qui

annonçaient autre que les orageons

et les misanthropes... )

Mais si...

Tu $n^{\prime}$ est pas sans ressource

Et de ton visage glacé

C'est bien et vide dans

son expression

que Darwin avait raisin

en dévignant que le

vin noble n'oublie pas l'eau de vie

$\mathbf{m}$ même quand 1 'île fait sec ...

'a M. grand Goutier et sa fame

les parens (these) de tout

plage qu'il y a.

BIBERONS. 